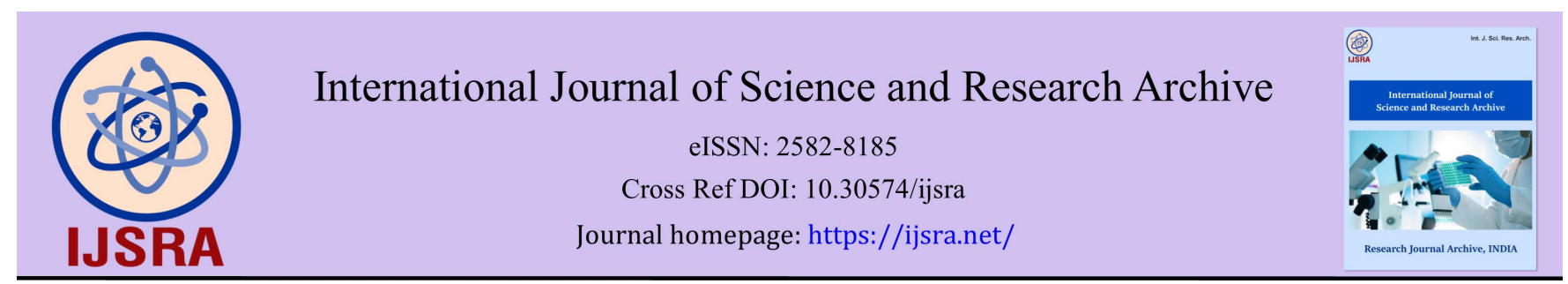

(RESEARCH ARTicle)

\title{
Leading role of the principles of interaction between the natural physical factors and integral organism in medicine of the future
}

\author{
Vladimir Dodtievich Bitsoev* \\ Academy of Medical and Engineering Sciences 3/1 Kasatkina Street, Moscow State Budget Healthcare Institution of \\ Moscow region "PTCH" Podolsk Town Clinical Hospital, 38, Kirova Street, Podolsk, Moscow region.
}

International Journal of Science and Research Archive, 2021, 02(01), 085-105

Publication history: Received on 10 January 2021; revised on 12 February 2021; accepted on 14 February 2021

Article DOI: https://doi.org/10.30574/ijsra.2021.2.1.0023

\begin{abstract}
The paper discusses the search of evidences of advantage of the physiotherapy over the drug therapy by the parameters and treatment methods according to clinical stage of a disease development, based on the results of labor medical examination and improvement of the quality of life.

The above indicators were confirmed with a high statistical accuracy by example of a new rehabilitation technology: "Underwater horizontal spine traction with underwater phototherapy to solve an essential medical and social problem: treatment of patients with degenerative and dystrophic lesions of the lumbosacral spine with herniated protrusions of intervertebral discs, being one of the most common neurological pathologies worldwide.

Based on the achievements in physics, i.e. discovery of "evanescent waves", "scanning tunneling microscope"; "AC and DC Josephson effects", development of highly sensitive equipment and discovery by the author of the present article of a highly informative, non-invasive, simple, harmless method of studying the mechanism of action of physical factors on the integral organism from any part of the skin surface, i.e. "blood spectrum biopsy" displaying the complete information picture of an organism, it became possible to create a new theory of a mechanism of action of physical factors on the supramolecular level of the integral organism on the principle of "tunnel effect".
\end{abstract}

Keywords: Physiodynamics; Physiokinetics; Supramolecular structures; Blood spectrum biopsy

\section{Introduction}

Any pharmacological preparation, from the moment of its introduction into organism and during the whole way of movement is exposed to complex physical and chemical changes with formation of reversible and irreversible compounds (intermediate formations) with obligatory release of a certain energy for continuation of cyclic process of transition from one state to the next one up to exit from the integral organism.

It is known that "the interaction of a medicinal product with the organism is studied in two aspects: how it affects the body (pharmacodynamics) and what happens to it in the body (pharmacokinetics). Pharmacodynamics studies localization, mechanism of action and pharmacological effects of the medicinal substances.

Pharmacokinetics studies the common factors of absorption, distribution and elimination of the medicinal substances in a human or animal organism. [1]

\footnotetext{
${ }^{*}$ Corresponding author: Vladimir Dodtievich Bitsoev

Academy of Medical and Engineering Sciences 3/1 Kasatkina Street, Moscow State Budget Healthcare Institution of Moscow region "PTCH" Podolsk Town Clinical Hospital, 38, Kirova Street, Podolsk, Moscow region. 
Herewith, it should be noted that the speed, scale, content and time of emergence of intermediate formations of pharmacological preparation in the organism are strictly individual for each patient.

The pharmacodynamics and pharmacokinetics of all medicinal products interacting with the body should be studied using quick, harmless and highly informative methods, before such products are accepted for medical use.

Compliance with this concept contributes to ensuring high therapeutic effect, primary and secondary prevention of diseases, prevention of complications and side effects on the body. This is not observed in global healthcare and pharmaceutical practice due to the lack of methods of research of pharmacological preparations at the supramolecular level.

From the organism's viewpoint, any substance coming from outside is regarded as a foreign body, thereby initiating a system of quick escape of the same. Even an own blood outside the vascular bed is treated as a foreign body by the organism. Therefore, an immediate shift of work of all organs and systems of the body into extraordinary mode takes place.

It should be noted, given the present state of development of the medicine, that there are no methods to determine the cycle temporal level of each system at the moment of effect of the medicinal substance on organism, in its transitional states at the supramolecular level and when all systems return to normal mode of life.

In this regard, it is difficult to imagine the advantage of a particular medicinal substance in general and, in particular, for a specific patient. The lack of data in the chronological sequence of the medicinal substance in its path to achieving the goal calls into question the prevalence of the drug therapy over physiotherapy.

It is known that the final characteristic of any medicinal substance at the supramolecular level is an "energy", which is difficult to dose and regulate for therapeutic purposes - this is the reason for the lack of these characteristics in the global medical and pharmaceutical literature.

The impact of energy of any physical factor is dosed and regulated (physiodynamics) with the help of nanotechnologies and its path to each molecule of the integral organism (physiokinetics) is easily traced without disturbing supramolecular structures and any negative consequences.

The modern global pharmaceutical science does not have such a high level of control over the path of a medicinal substance in the body.

According to A.L. Chizhevsky "an obligatory condition for the life of a living organism is an exchange of the "electrical" component being the foundation of metabolism process, and the total energy content in the cellular structures of the body has a strictly defined meaning". [2]

Bioelectric potential for each human being is strictly individual both in norm and pathology. In this regard, any nosology causes in each individual a deviation of his or her bioelectric potential in accordance with the stage of disease development, i.e. development of intermediate states of the body with certain disorders of its supramolecular structures.

This, in turn, determines the clinical findings at the time of examination of the patient and constitutes the leading condition for selecting the right treatment strategy for any specialist physician, so that the regression of the disease is accompanied by the restoration of destroyed supramolecular structures, avoiding new gross disturbances at any level of the integral organism.

This concept is not the main principle for drug therapy due to the lack of highly informative research methods: frequency, dose, mechanism of action of pharmacological preparations at the supramolecular level. 


\section{Materials and methods}

\subsection{Experimental studies}

Methods of experimental study of the action of PVIP light device "BIOPTRON" via fiber optic cable to water, blood plasma and integral organism: IR spectroscopy in the region $4000-400 \mathrm{~cm}^{-1}$ on a Fourier spectrometer Perkin-Elmer 2000 between the plates KRS-5, conducted at the Establishment of the Academy of Science A.A. Frumkin Institute of Physical Chemistry and Electrochemistry of the Russian Academy of Science ( IPCE of RAS), Moscow; Experimental measurements of the spectra of Raman scattering (Raman effect) of various water samples on the automated fiber optic spectrometers at the Institute of Spectroscopy of the Russian Academy of Sciences RAS (ISAS), town Troitsk, Moscow region and the Research Center of fiber optics of the Russian Academy of Sciences, Moscow; Evanescent infrared spectroscopy of the skin in vivo by fiber optic sensor, at the Scientific center of Fiber Optics of the Russian Academy of Sciences, Moscow.

\subsection{Clinical trials}

This paper is based on analysis of the treatment outcomes of 745 individuals (454 males and 291 females) aged 17 to 60 years with degenerative and dystrophic processes of intervertebral discs of lumbosacral spine, with hernial protrusions into the spinal canal of up to $13 \mathrm{~mm}$.

In accordance with the purpose and tasks of the trial, all patients were examined and splitted up into groups according to following indices:

Four age subgroups: 1) aged 17 to 30 years; 2) 31 to 40 years; 3) 41 to 50 years; 4) 51 to 60 years) and by gender (males and females);

By duration of the disease ( $n=745): 1) 1$ to 5 years; 2) 6 to 10 years; 3 ) more than 10 years;

By incidence of various clinical evidences of degenerative and dystrophic processes in lumbosacral spine ( $\mathrm{n}=745)$;

By quantitative characteristic of radiodiagnostic data: computer tomography (CT) - 244, magnetic resonance imaging (MRI) - 269, X-ray radiography - 745;

By MRI results and age groups of randomized trial population $n=269$ with hernial protrusion into the spinal canal $L 4$ $\mathrm{S} 1$ of up to $5 \mathrm{~mm}$ and from 5 to $13 \mathrm{~mm}$;

All of the patients were splitted up into 5 groups by treatment methods and into 4 groups by age.

$1^{\text {st }}$ control group - 175 patients: 24 patients aged 17 to 30 years, 37 patients aged 31 to 40 years, 65 patients aged 41 to 50 years, 49 patients aged 51 to 60 years, were administered the drug therapy according to Moscow standard. Agerage therapy regimen was 20 days.

$2^{\text {nd }}$ control group - 157 patients: 26 patients aged 17 to 30 years, 39 patients aged 31 to 40 years, 62 patients aged 41 to 50 years, 30 patients aged 51 to 60 years were administered the physiotherapy (magnetotherapy, amplipulse therapy, laser therapy, acupuncture, ultrasonic therapy, therapeutic physical training in various combinations) with drug therapy.

Average therapy regimen was 17 days. Information for each patient was collected according to medical documents (outpatient records, medical and physiotherapeutic treatment procedural records), registers of primary and recurrent patients, registers of specialists), as well as by interviews and questionnaires according to the "map of examination for detection of musculoskeletal pathology" (Appendix 1) developed by us, which represents a slightly modified standard WHO questionnaire.

The rest of 413 patients under attendance were distributed into three groups by the adopted treatment methods and into four age groups, the same as two control groups: 
$3^{\text {rd }}$ group: 26 patients aged 17 to 30 years, 39 patients aged 31 to 40 years, 55 patients aged 41 to 50 years, 33 patients aged 51 to 60 years, a total of 153 patients were administered the physiotherapy with underwater phototherapy with the average treatment regimen of 16 days.

$4^{\text {th }}$ group: 26 patients aged 17 to 30 years, 32 patients aged 31 to 40 years, 43 patients aged 41 to 50 years, 31 patients aged 51 to 60 years, a total of 132 patients were administered the physiotherapy with underwater spine traction. Average treatment regimen was 15 days.

$5^{\text {th }}$ group: 30 patients aged 17 to 30 years, 30 patients aged 31 to 40 years, 45 patients aged 41 to 50 years, 23 patients aged 51 to 60 years, a total of 128 patients were administered the underwater spine traction with underwater phototherapy. Average treatment regimen was 16 days.

\section{Results and discussion}

Clinical physiotherapy is closely connected with the other fields of the medicine, physics and biology.

Thus, the investigation of etiology and pathogenesis of many diseases allows not only the application of the necessary physical factor, but the development of a rational method, its application, combination with other physical factors as well $[3,4,5,6]$.

The achievements in physics and development of highly sensitive equipment made possible determination of the mechanism of action of low-energy physical factors on cellular and subcellular levels of the organism [7,8,9].

It is established that when any electromagnetic wave passes through the fiber, evanescent waves are always formed around the fiber perpendicular to the outer surface of the latter and they attenuate at a distance from it.

These previously unknown light properties were recently discovered by scientists from Japan, USA, Ukraine and Korea [10].

In this regard, we have conducted a number of experimental studies (recording of standing evanescent wave photons on the skin surface after exposure to PVIP (Polychromatic Visible and Infrared Polarized) light of 480-3400 nm).

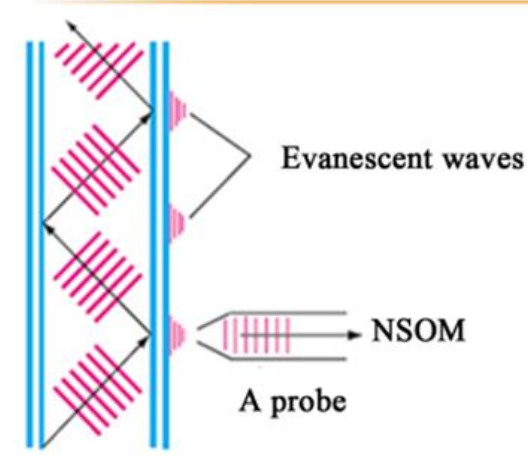

A light guide with a wave advancing along it

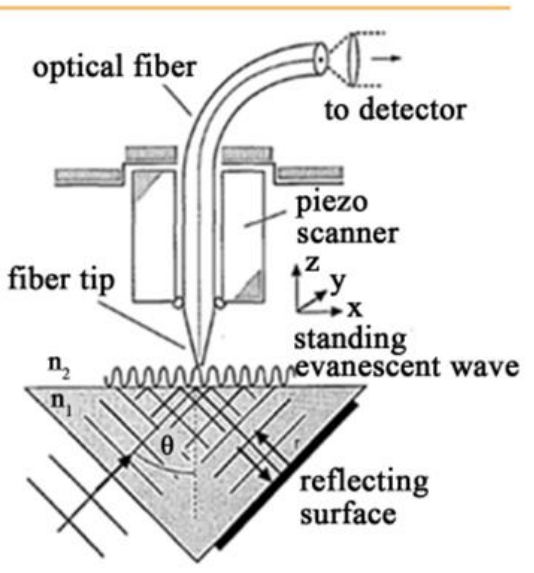

Diagram of registering photons of a standing evanescent wave bonded with top prism surface using a scanning tunneling photon microscope.

Figure 1 Evanescent waves 


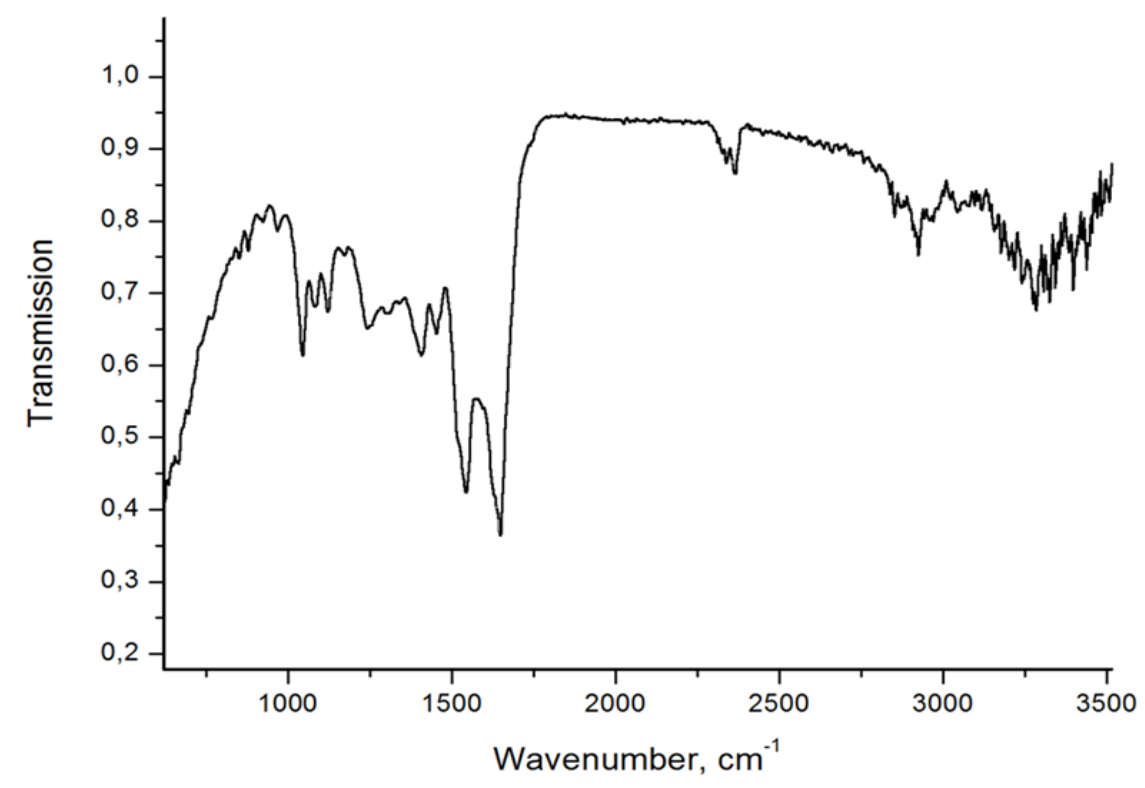

Figure 2 IR spectrum of the skin after hand exposure to yellow light through the optic fiber cable during ten minutes.

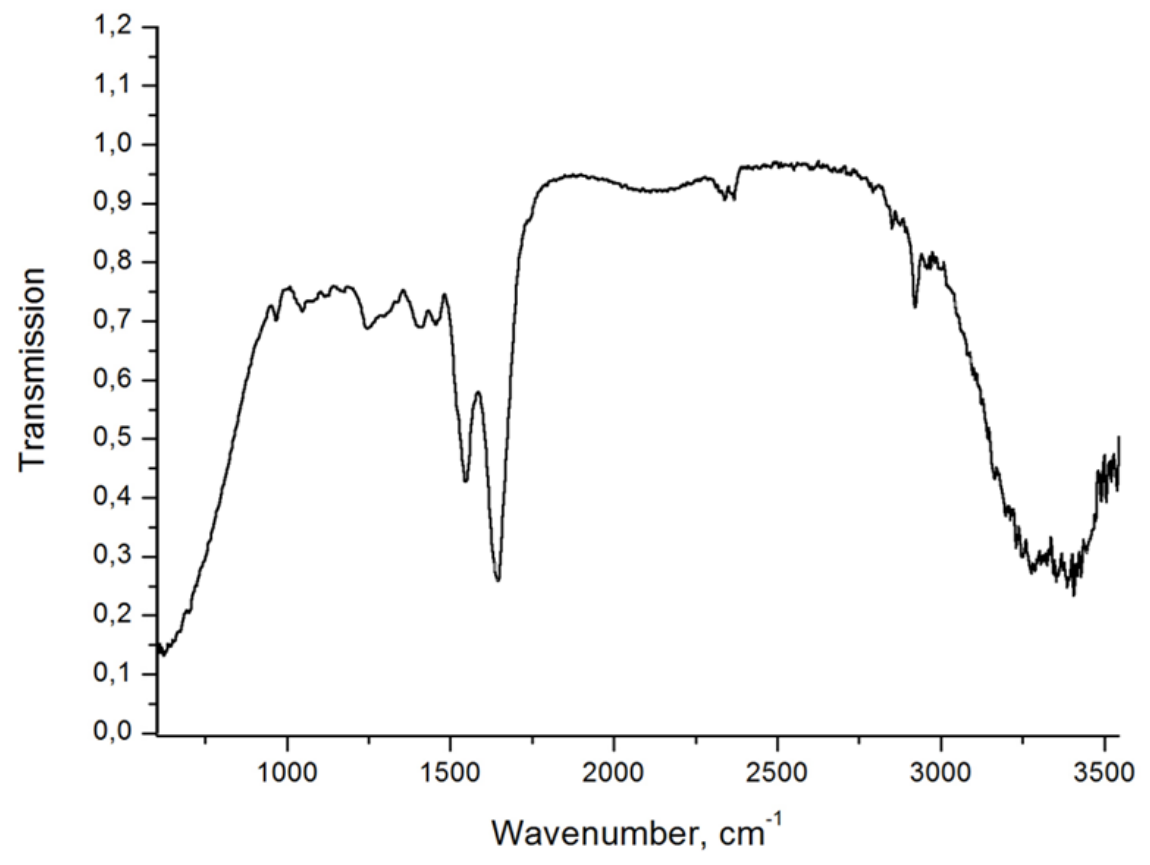

Figure 3 IR spectrum of the skin after hand immersion into the water irradiated by yellow light through the optic fiber cable during ten minutes. 


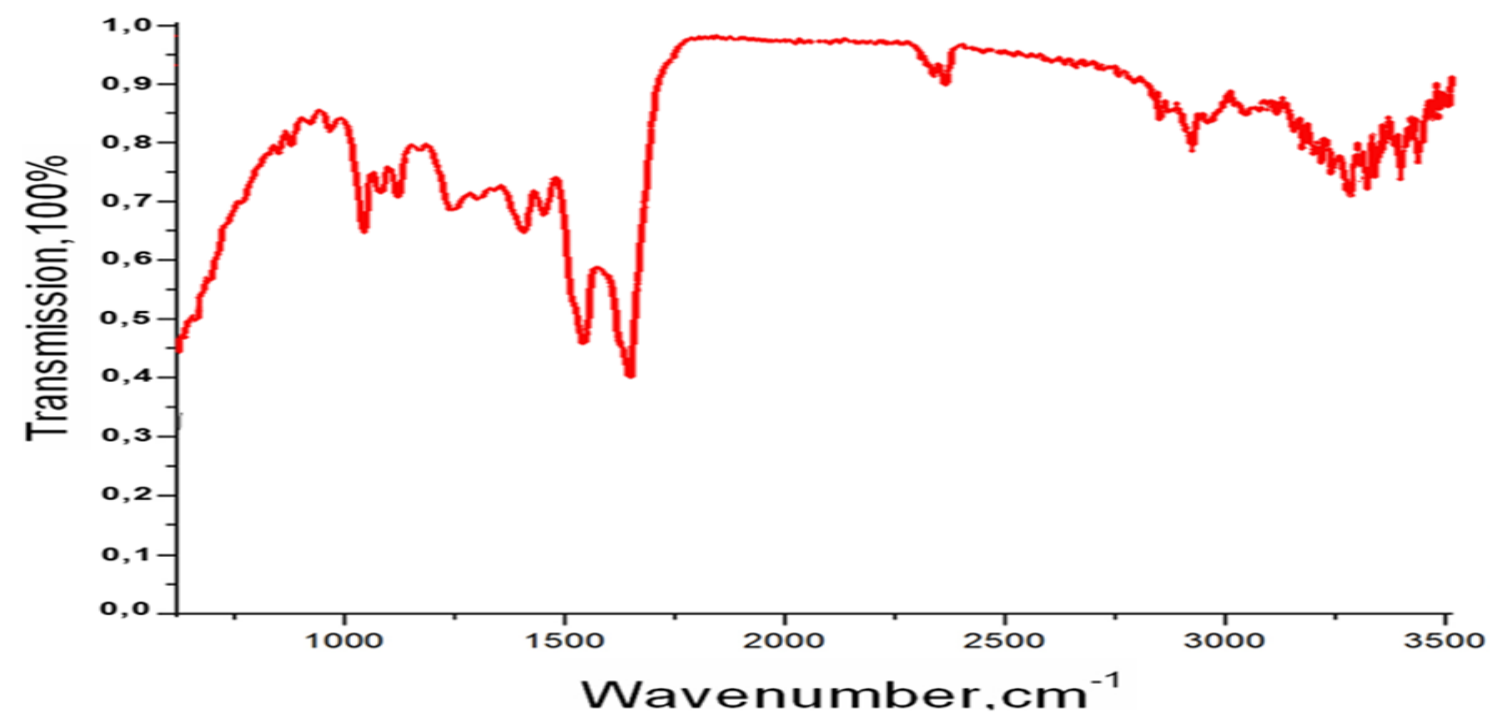

Figure 4 IR spectrum of the skin after hand exposure to polarized light emitted by Bioptron apparatus during 10 minutes.

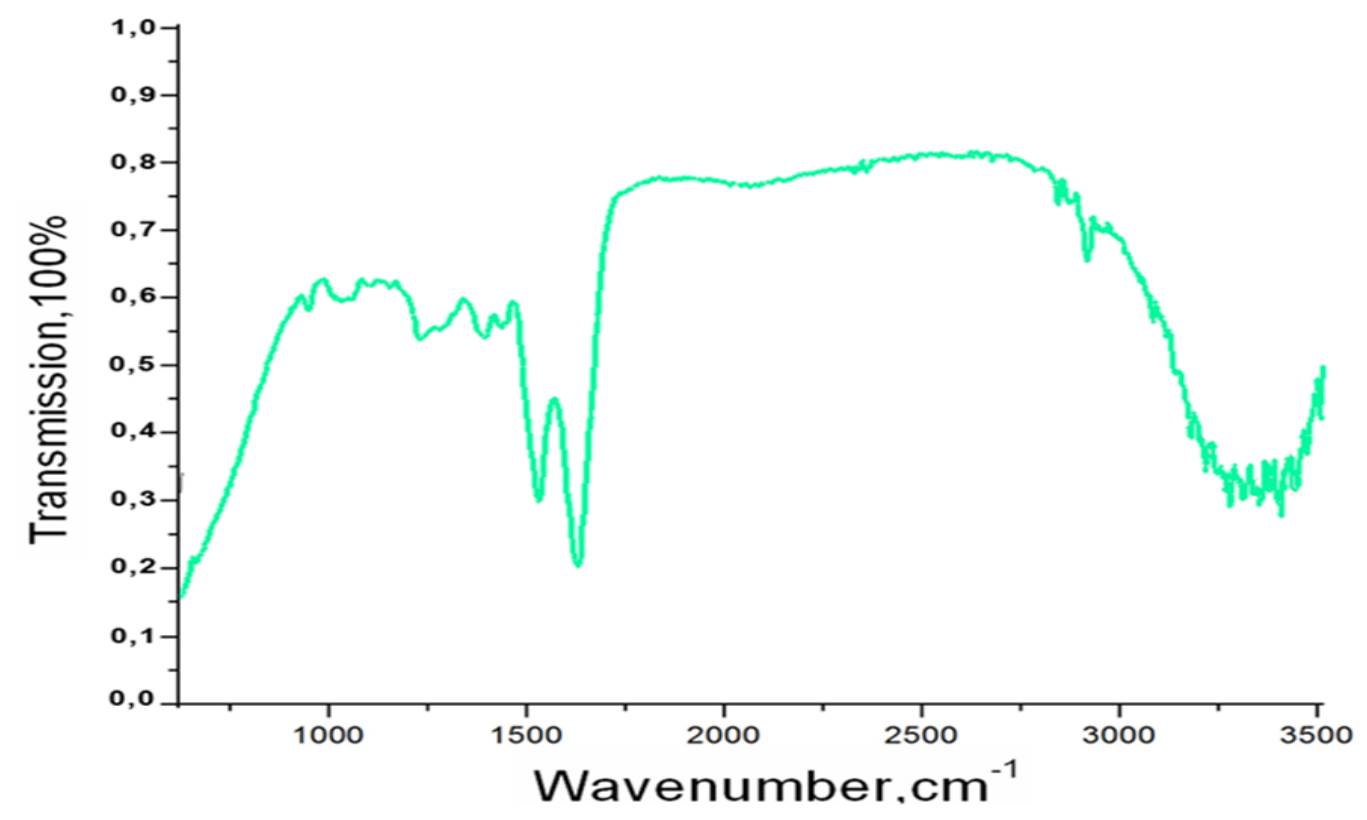

Figure 5 IR spectrum of the skin after hand immersion into the water irradiated by Bioptron emitted light during 10 minutes.

It was established that the evanescent infrared spectra of the skin after exposure to water activated by light through the fiber optic cable from Bioptron device demonstrate an increase in the absorption area within the wavelength ranges from 3200 to $3500 \mathrm{~cm}^{-1}$ and within the range up to $800 \mathrm{~cm}^{-1}$, i.e. there is an increase in hydration [11,12,13,14].

In this regard, there is a substantiation to use phototherapy with Bioptron device through fiber optic cable both under direct exposure and indirectly, i.e. through water, for treatment of various diseases of the musculoskeletal system, skin and other organs and systems.

Based on the above experiments, it is possible to conclude that the photoexcitation of water by Bioptron source indeed results in changes of water structure. This was made possible by absorbing the radiation from the source in the water, stimulating the growth of water nanoclusters linked by hydrogen bond, which, in its turn, was made possible by discovering the unusual properties of light by the abovementioned scientists (emergence of evanescent waves) and creation of "scanning tunneling microscope". (Dr. Stephen Mendak and his colleagues from Germany created a working 
tool able to reconstruct two-dimensional images of three-dimensional nanoscale objects using the light of visible frequency and the near infrared spectrum. The device was developed on the basis of the "super lenses" made of thin silver strips in the form of a tube with center opening of about $2 \mu \mathrm{m}$. The evanescent waves pass from its inner surface perpendicularly to the circumference and during such movement there is a primary image magnification, i.e. the "scanning tunneling microscope") [10]

Russian scientist, winner of the Lenin Prize in physics Gurgen Ashotovich Askarian in 1982 experimentally proved: "increasing in the passage of laser and other radiation through soft turbid physical and biological environments" [15].

It is known that one of the interesting applications of laser is based on its stimulating effect on regenerative, antiinflammatory and immune processes in cells and tissues of the body, especially in view of the resonant nature of absorption and stimulation (one of the absorption fields is close to the generation line of a He-Ne- laser with a wavelength of $0.63 \mu \mathrm{m})$.

It is precisely these processes that link the use of such lasers with the treatment of purulent processes of the maxillary sinuses, trophic ulcers, non-healing wounds, stomatitis, polyneuritis and other pathological processes.

In this regard, we widely used low-energy lasers in the comprehensive therapy of trophic long-term non-healing wounds, on an annual basis, for 150-170 patients in average, according to the following method:

Magnetotherapy of a limb during 5-7 minutes up to $30 \mathrm{mt}$, transversely to the wound area during 5-7 minutes up to 30 mt (Polyus-1 device);

Ultratone therapy (supersonic frequency currents) on the wound area, average power, during 10 minutes;

Laser therapy: 4 areas around the wound perimeter, stably for 4 minutes each, $1500 \mathrm{~Hz}$ and in the fifth area in the wound center, stably for 4 minutes, $80 \mathrm{~Hz}$.

The treatment regimen consisted of 17 procedures.

There was always a persistent remission and complete healing of the wound.

Over the past five years, an average of 180 patients with the diagnosis of "bilateral purulent process of the maxillary sinuses" were treated annually by physiotherapy according to the following profile:

UHF, maxillary sinus area $40 \mathrm{~W}, 10$ minutes, treatment regimen of 10 days;

Irradiation of nasal passages with short-wave ultraviolet rays for 40 seconds, 10 days;

Laser therapy, area of the maxillary sinuses with moderate pressure of the device Uzor-A-2K emitters of soft tissues over the areas of the nasal sinuses, $5 \mathrm{~Hz}$, for 5 minutes, treatment regimen of 10 days.

There was always a clinical recovery under the X-ray radiography monitoring of the paranasal sinuses and patients did not need surgical treatment.

G. A. Askaryan used in his first experiment a layer of polyurethane foam simulating a highly scattering medium. He used as an experiment a continuously operating He-Ne laser LG-75 with a power of 15-20 mW. Its beam was aimed at the test layer contained by a cylinder or tube. A spot of the scattered light was recorded by the camera at the exit from the scattering layer. The spot of scattering light at the exit depended on thickness of the compressed layer. The lower the thickness of the compressed layer was, the larger was the spot of the scattered light at the exit [15]. 

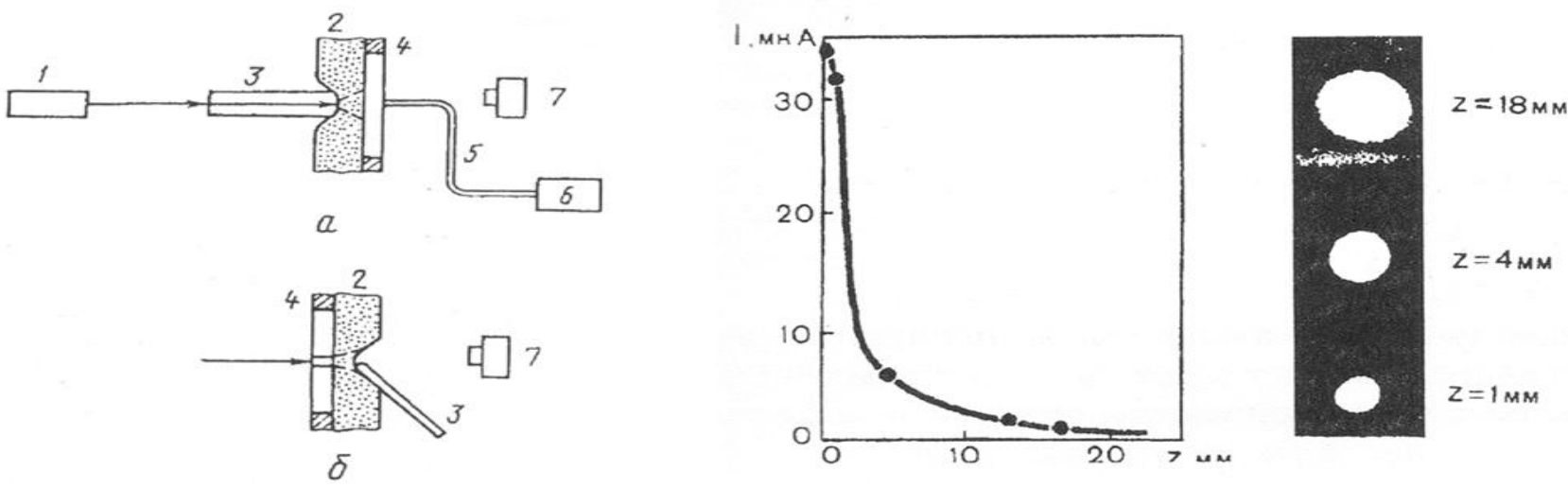

Figure 6 Increased light penetration when pressing on the foam layer

In his second experiment, G. A. Askarian investigated the change in the light penetration through tissues of the human body. He used a palm as a scattering layer, the center of which was hit by a helium-neon laser beam. The thickness of the palm in its central part was $2.7 \mathrm{~cm}$ and $2 \mathrm{~cm}$ with slight pain compression.

When the beam hit the back of the right palm and when the glass stick pressed on the flesh of the palm from the opposite side, a sharp increase in the light passage was observed. When pressing the left palm on the plexiglass cylinder through which the light passed, the increased light passage observed from the opposite side. Such increase was observed already when compressing from 2.7 to $2 \mathrm{~cm}$, i.e. the translucence effect is much more intense when pressing the palm than when using the foam [15].
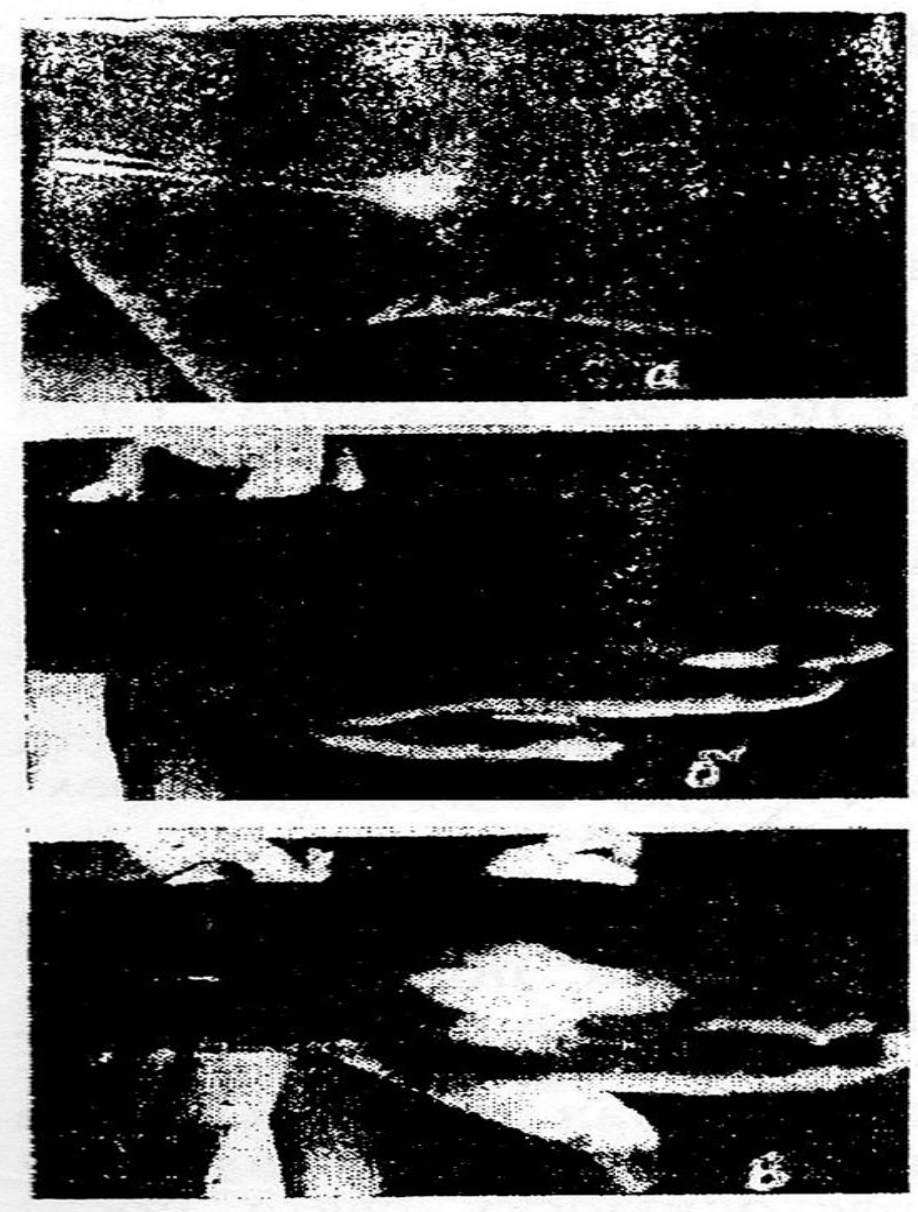

Figure 7 Increased light penetration when pressing on the biologic body layer. 
The author attributes this to the lateral displacement of blood and tissue. However, it should be noted in his experiment that even the thicker parts of the palm, i.e. the flesh with blood and area at the phalanges of fingers are more permeable to light and more susceptible to translucence by compression than its central part. It is interesting to note the relaxation of translucence: when the pressure is released, the translucence does not disappear immediately - the trace of pressure passes the light for another 1 to 3 seconds. This is confirmed in papers [11,12] - irradiation aftereffect.

The works $[12,13,14,16]$ established that the cardiovascular, nervous and lymphatic systems can be the light guides and evanescent (attenuating) waves also form around them, perpendicular to the outer surface of vessels, capillaries, and nerve roots, when they are exposed to electromagnetic waves in any areas of the skin surface.

In this regard, we have developed a sensor-mininanomicroscope, through which a direct and back communication of internal organs and systems of the integral organism is carried out.

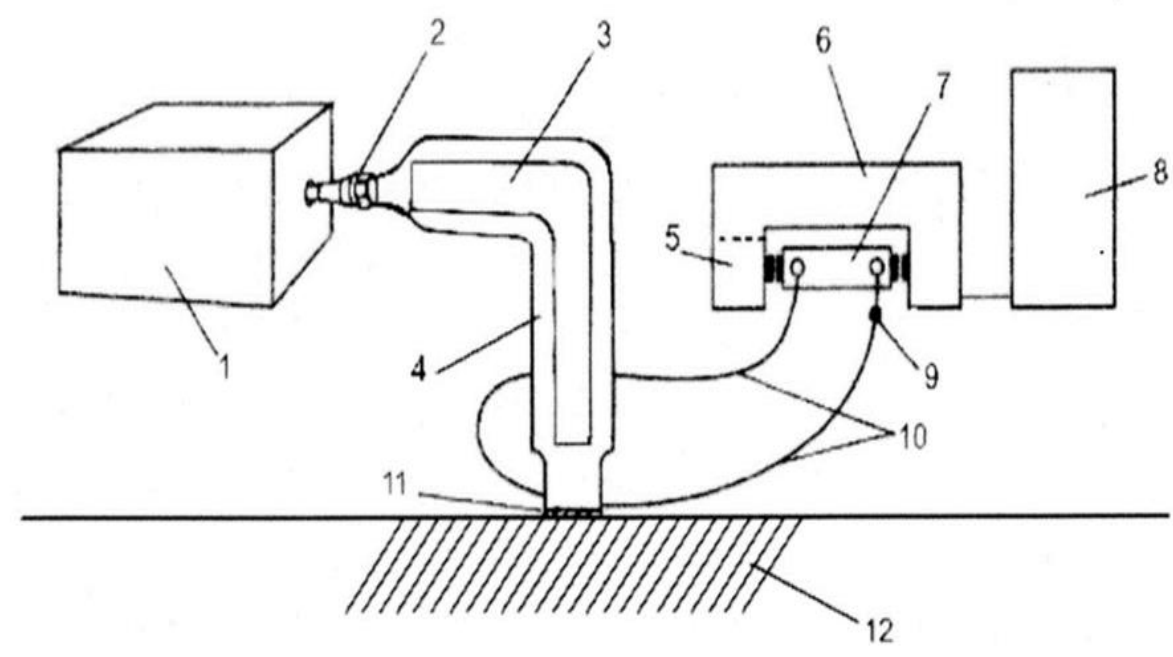

Figure 8 Recording of direct and back communication of all organs and systems of the integral organism from the skin surface.

1. Light source.

2. Coupling head inserted into the opening of the metal box (connects the source of light and optical fiber cable).

3. Optical fiber cable.

4. Device of information exchange between the integral organism and biosphere. (Figure 8(a))

5. DTGS pyroelectric detector

6. Spectrometer Bruker «Vector 22».

7. The mirror-lens accessory for connection of "Device of information exchange between the integral organism and biosphere" to Fourier-transform spectrometer "Vector 22".

8. Computer.

9. Contact switch.

10. Terminals from the shell-less fiber AgClBr.

11. Sensor-mininanomicroscope. (Figure 8(b))

12. Skin surface of the integral organism. 


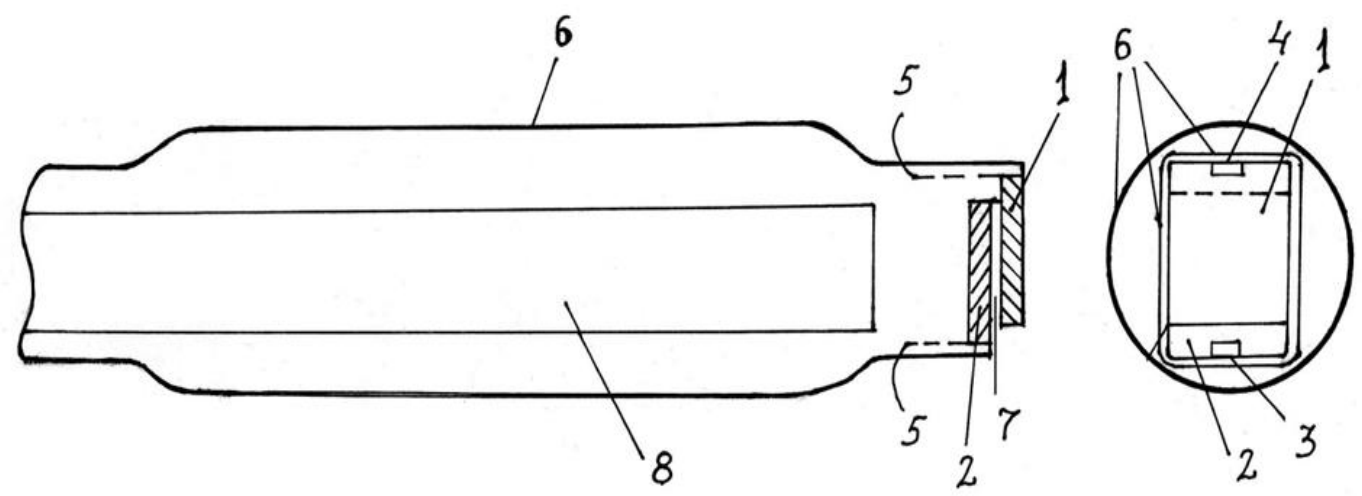

Figure 8(a)

1. Upper glass plate

2. Lower glass plate

3. Silver plate

4. Silver plate

5. Terminals.

6. Flexible non-metallic tube

7. The space between the skin surface of the skin of a living organism and the upper glass plate with a transition to the space between two glass plates

8. Optical fiber cable

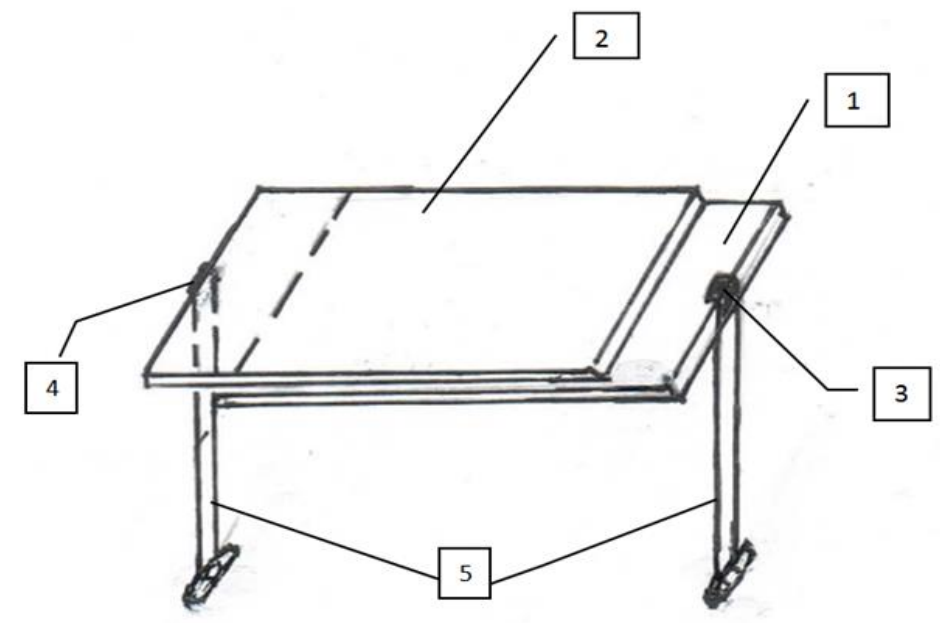

Figure 8 (b)

1. Upper glass plate.

2. Lower glass plate.

3. Silver contact plate.

4. Silver contact plate.

5. Terminals from the silver plate

The mininanomicroscope sensor is made of two glass plates, placed one above each other with displacement of $5 \mathrm{~mm}$, as to provide space between the skin epidermis and upper glass plate, as well as of $7 \mu \mathrm{m}$ space between the glass plates for information reciprocal exchange between the integral organism and biosphere through the perspiration system and optical fiber cable. 
Therefore, evanescent IR spectroscopy of the attenuated total reflection when touching the IR fiber us an unique, nondamaging, not requiring special preparation of the skin method - "Blood Spectrum Biopsy", which reflects the entire information picture of the body at the supramolecular (atomic) level (Fig. 2, 3, 4, 5, 6, 7, 9, 10, 11, 12, 13,).

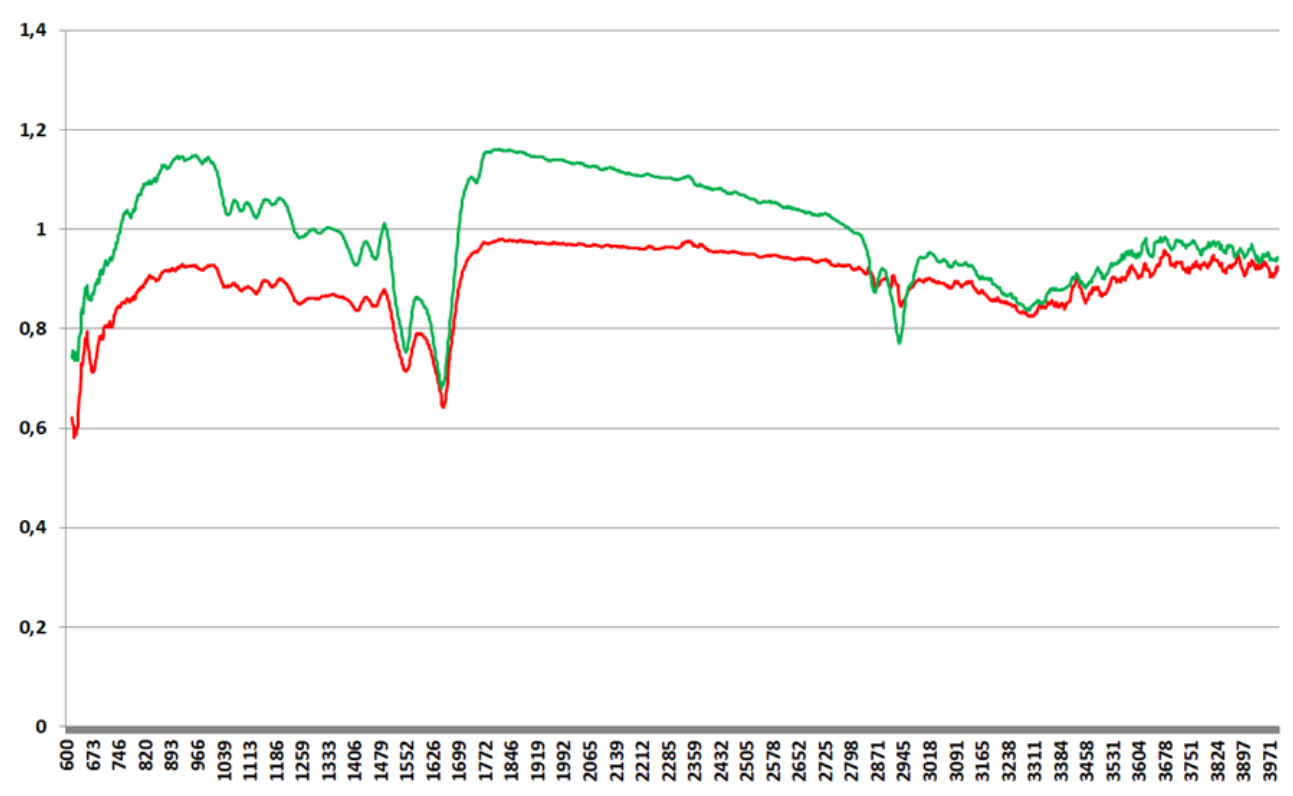

\section{Palmar surface}

Back surface

Figure 9 Blood spectrum biopsy with non-irradiated surface of the palm.

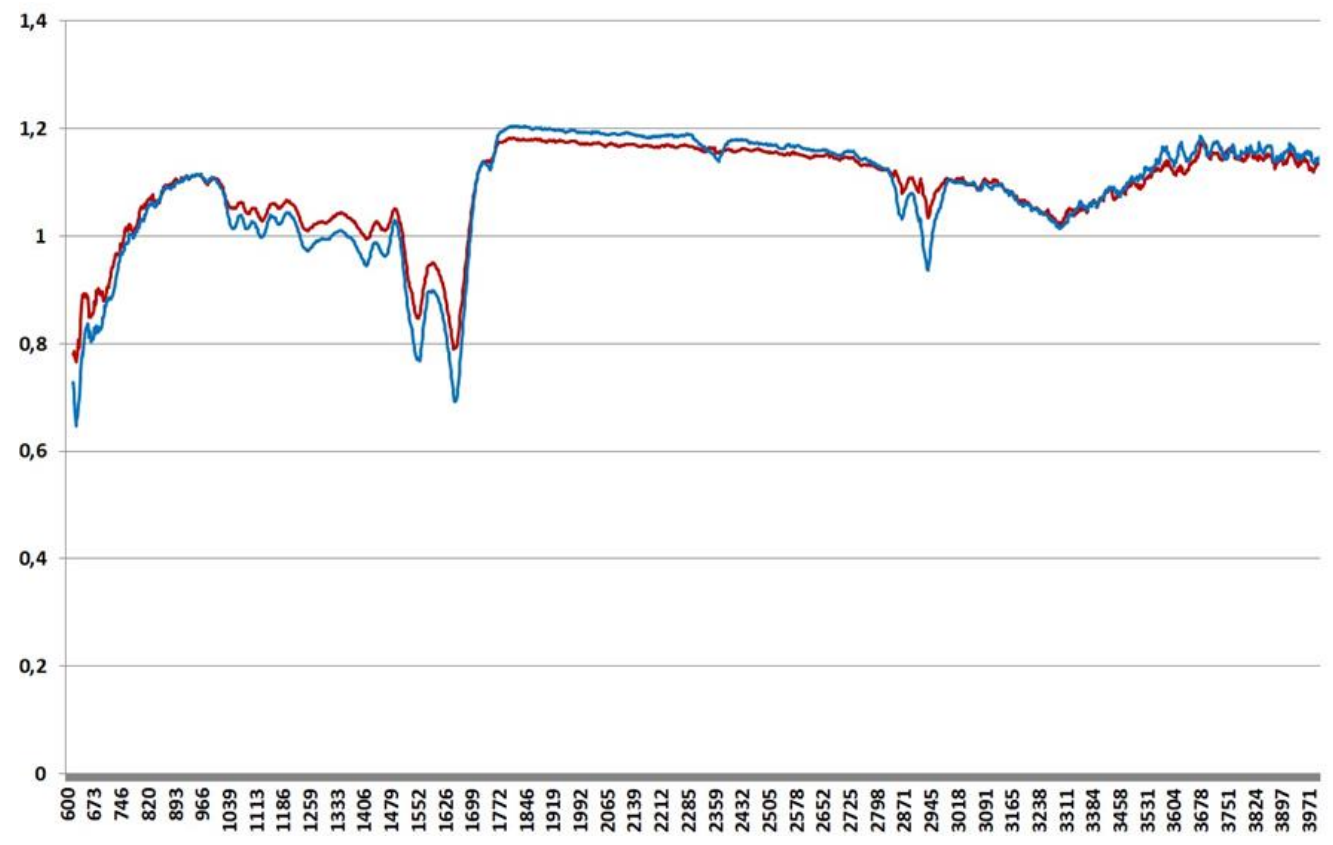

Palmar surface

Back surface

Figure 10 Blood spectrum biopsy from the palm surface irradiated by yellow light during $10 \mathrm{~min}$ 


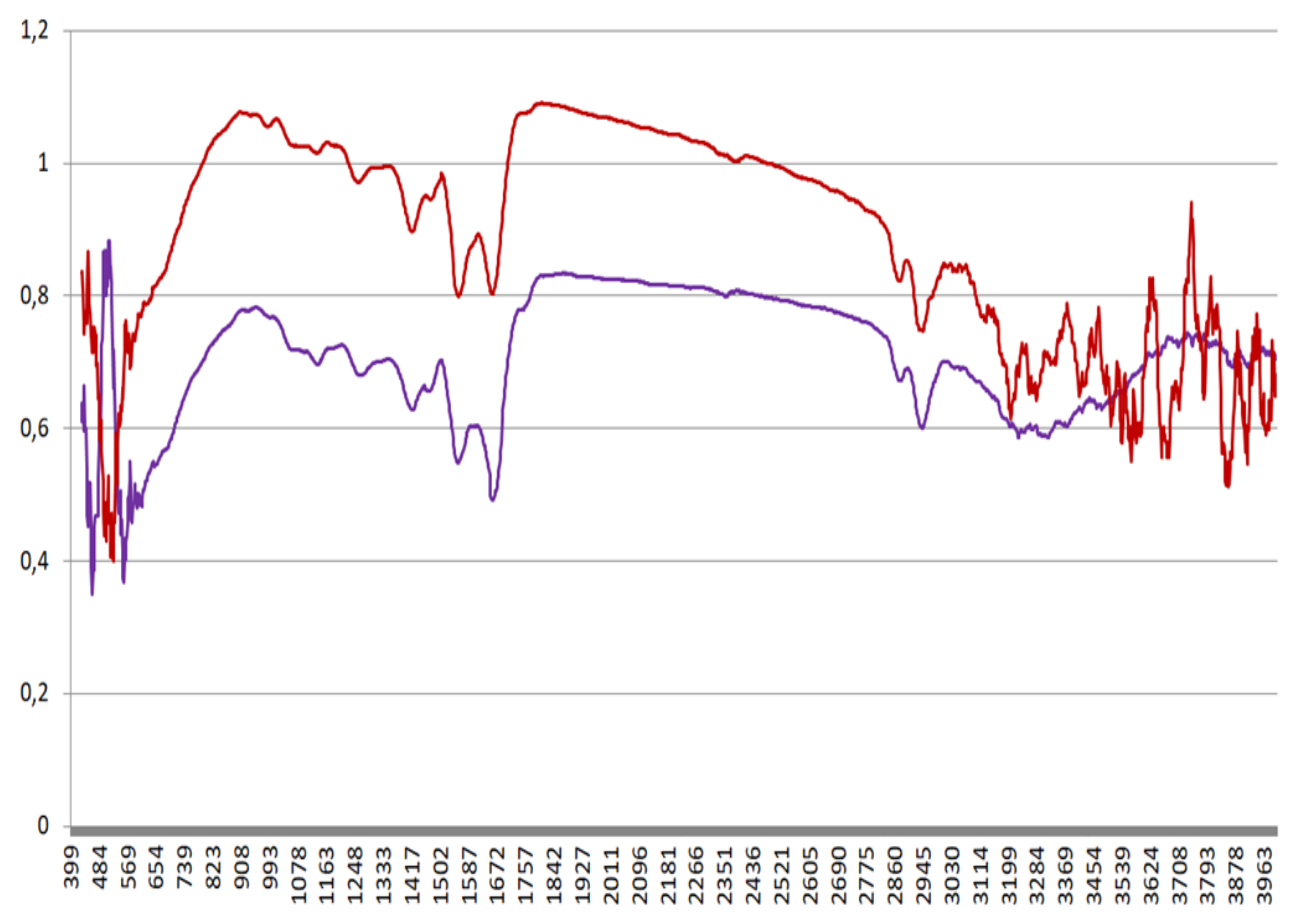

Palm surface after removal of two layers with adhesive tape

Palm surface irradiated by yellow light during 10 minutes.

Figure 11 Blood spectrum biopsy from the hand surface.

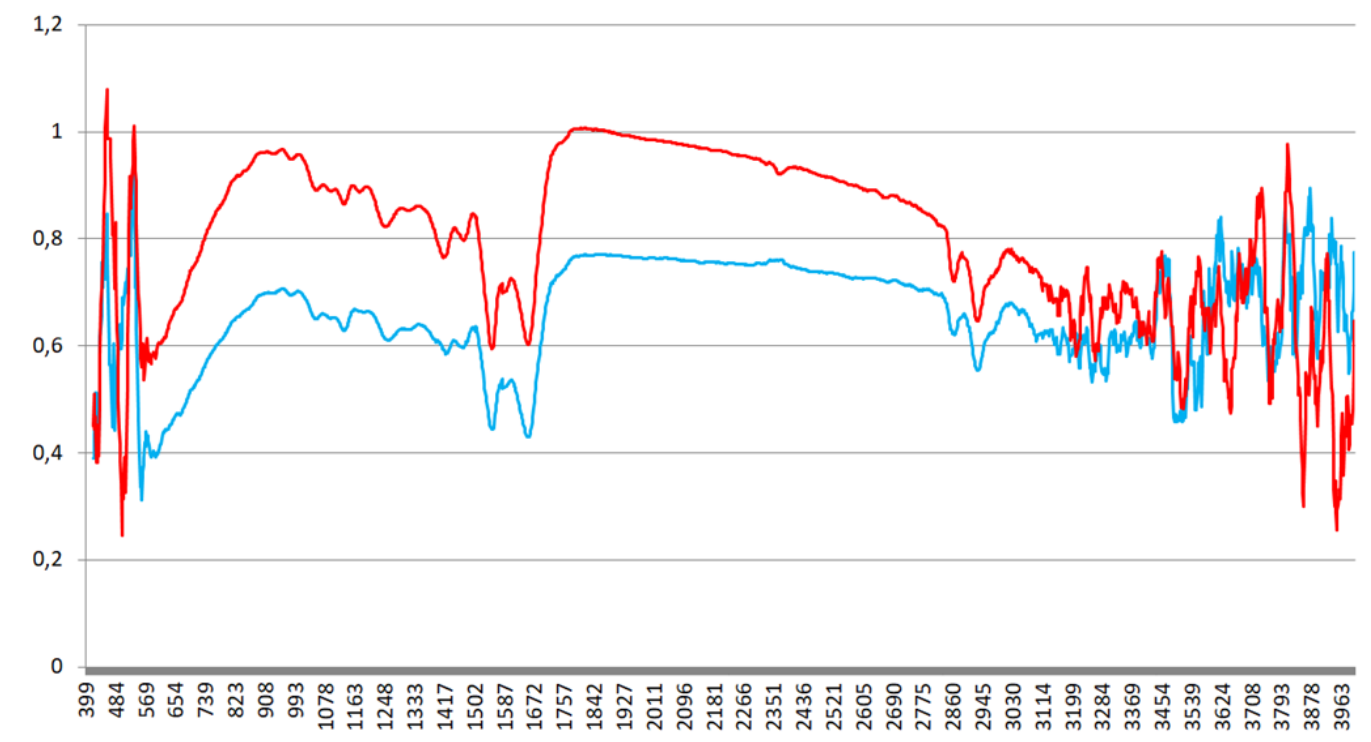

Back surface after removal of two layers of skin with the adhesive tape

Back surface of the hand irradiated during 10 minutes.

Figure 12 Blood spectrum biopsy from the hand surface. 


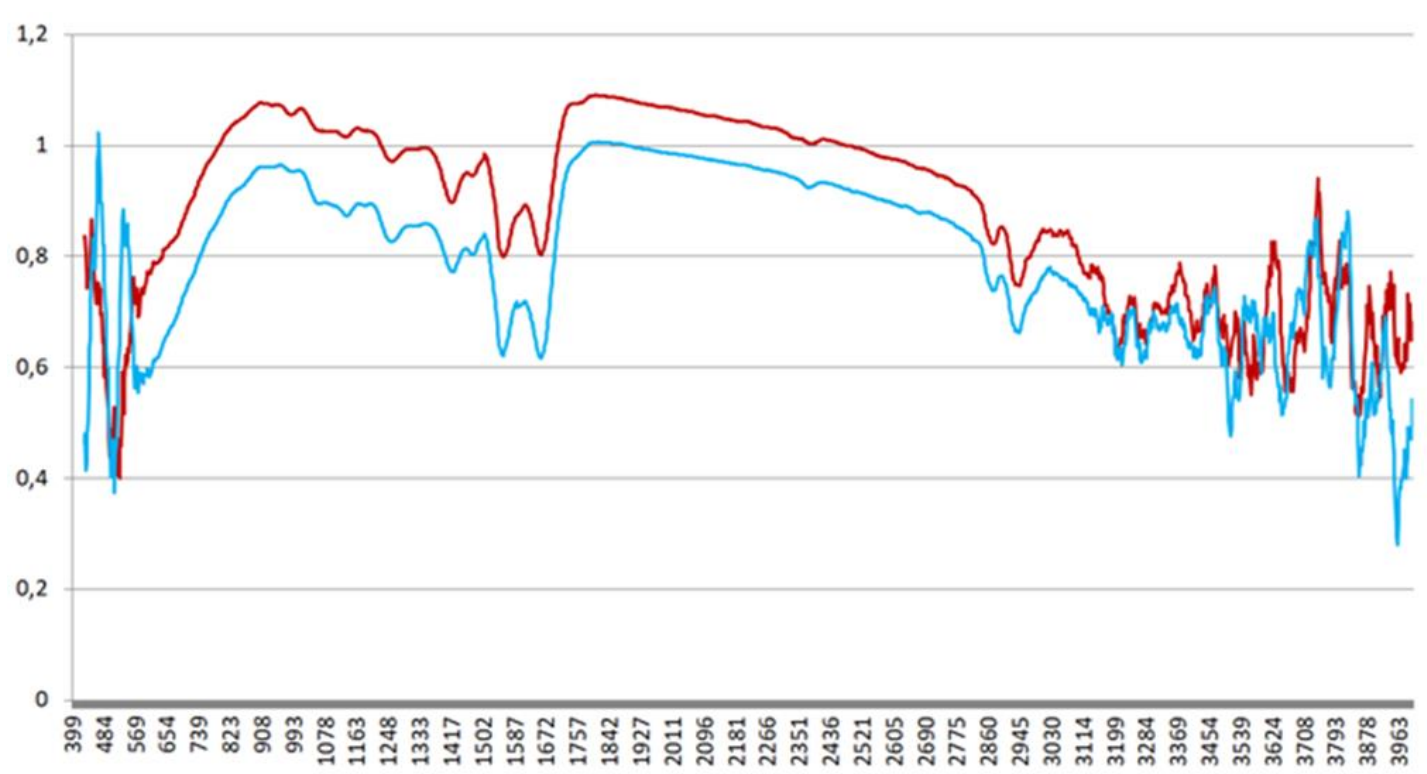

Palm surface after removing two layers of skin by adhesive tape, irradiated by yellow light during 10 minutes. Back surface after removing two layers of skin by adhesive tape, irradiated by yellow light during 10 minutes.

Figure 13 Blood spectrum biopsy from the hand surface.

Based on the results of experiments, we can assert that the experiments of G. A. Askarian of "increase in the passage of laser and other radiation through turbid physical and biological mediums" support our concept that an integral organism reacts to any external influence on the principle of "biological scanning tunneling microscope", i.e. the light passage through the entire thickness of palm is not due to the lateral displacement of blood and tissue, as stated by the author of the experiment, but to a layer-by-layer formation of evanescent waves deep in the palm with their subsequent increase at the exit of the opposite side of the palm. Also in favor of our concept, the conclusion of the author of the experiment is that the effect of illumination when pressing the palm is much stronger than when using polyurethane foam, and even the thicker parts of the palm - the flesh with blood, the area at the phalanges of the fingers is more permeable to light and more susceptible to illumination by compression than its central part.

Our concept of the tunnel effect of the integral organism emerging at exposure to low-energy electromagnetic waves is supported by the works of Russian and foreign scientists. They discovered the causes of emergence of the evanescent waves: Doctor of Physics and Mathematics, Professor German Nikolaevich Zhizhin, Doctor of Physics and Mathematics, Professor Vinogradov , V. N. Galynsky, A. I. Furs, L. M. Barkovsky, and some others. They believe that the interaction between photons and elementary excitations of the medium produces polaritons. The interaction between electromagnetic waves and excitations of the medium (phonons) leading them to bond becomes especially strong when their frequencies and wave-number vectors coincide (resonance). The bound waves are formed in this region, i.e. polaritons at the border of two media, and attenuate exponentially with the distance from the interface (near field). Phonon is a quasi particle introduced by the Russian scientist Igor Tamm representing a quantum of vibrational motion of crystal atoms.

Scientists discovered pseudo-particles traveling on the surface of light-sensitive materials.

Researchers from the Karlsruhe Institute of Technology, working together with scientists from the Fritz Haber Institute, Berlin, Germany, and Aalto University, Helsinki, Finland made a significant step towards implementing technologies for converting light into energy that can be used for the benefit of people [2,7].

Processes that convert light energy into energy of other types can and are gradually becoming the basis of technologies that will supply humanity with energy in the near future.

"The conversion of the energy of photons, particles of light, into electrical energy takes place in several stages," explains Professor Christoph Well, Head of the IFG Institute. First, light is absorbed on the surface of the light-sensitive material. The electrons leave their places as influenced by the energy of photons of light, leaving electron holes in their place, with which they immediately form quasi particles called polaritons. These polaritons exist only for a very short time, 
moving to the boundaries of the material, where they disintegrate into electrons and holes, which continue to move independently further on. The afterlife of these charge carriers already depends on the nature of the light-sensitive material used" [18].

It is known that the English physicist B. Josephson predicted in 1962 the DC and AC effects in the superconductordielectric contact on the basis of the Bardeen-Cooper-Schrieffer superconductivity theory.

Two superconducting layers separated by a negligibly thin insulator layer with thickness of a few atoms only will behave as a single system.

When a current is passed through the contact that does not exceed the critical value, there is no voltage drop on the contact (despite the presence of a dielectric layer).

This effect is caused by the fact that the conduction electrons pass through the dielectric without resistance due to the tunnel effect. Electrons can pass the barrier even if there is no voltage applied to them (Cooper pair tunneling). The effect is called the DC Josephson effect [19].

If a constant voltage is applied on both sides of the passage, quantum mechanics predicts that Cooper pairs of electrons will move through the barrier first in one direction and then in the opposite direction, resulting in an alternating current, which frequency increases as the voltage grows. This effect is called the AC Josephson effect" [18,19].

According to the laws of physics, when an integral organism is exposed to electromagnetic waves, evanescent waves are formed from the outside of all capillaries, vessels, nerve trunks and roots, this is a set of "scanning tunneling biomicroscopes", and the effect of PVIP light on the organism is recorded using a "scanning tunneling photon microscope". Therefore, it should be considered that the animal organism is a single superconducting system working on the principle of the Josephson effect in superconductors.

In this regard, there is reason to assert that we have identified new, previously unknown mechanisms of action of weak electromagnetic waves on the integral organism and ways to record the organism's responses. In other words, the integral organism functions on the principle of a "scanning tunneling near-field biomicroscope", as it corresponds to the description of the device by Stephen Mendek from Germany.

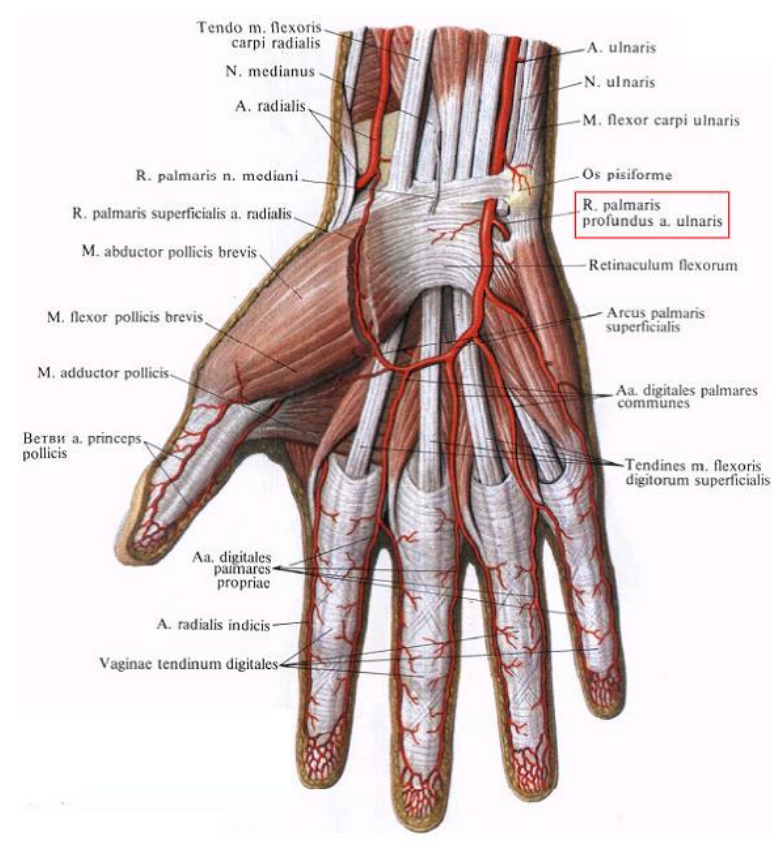

Figure 14 Arteries of right hand. 


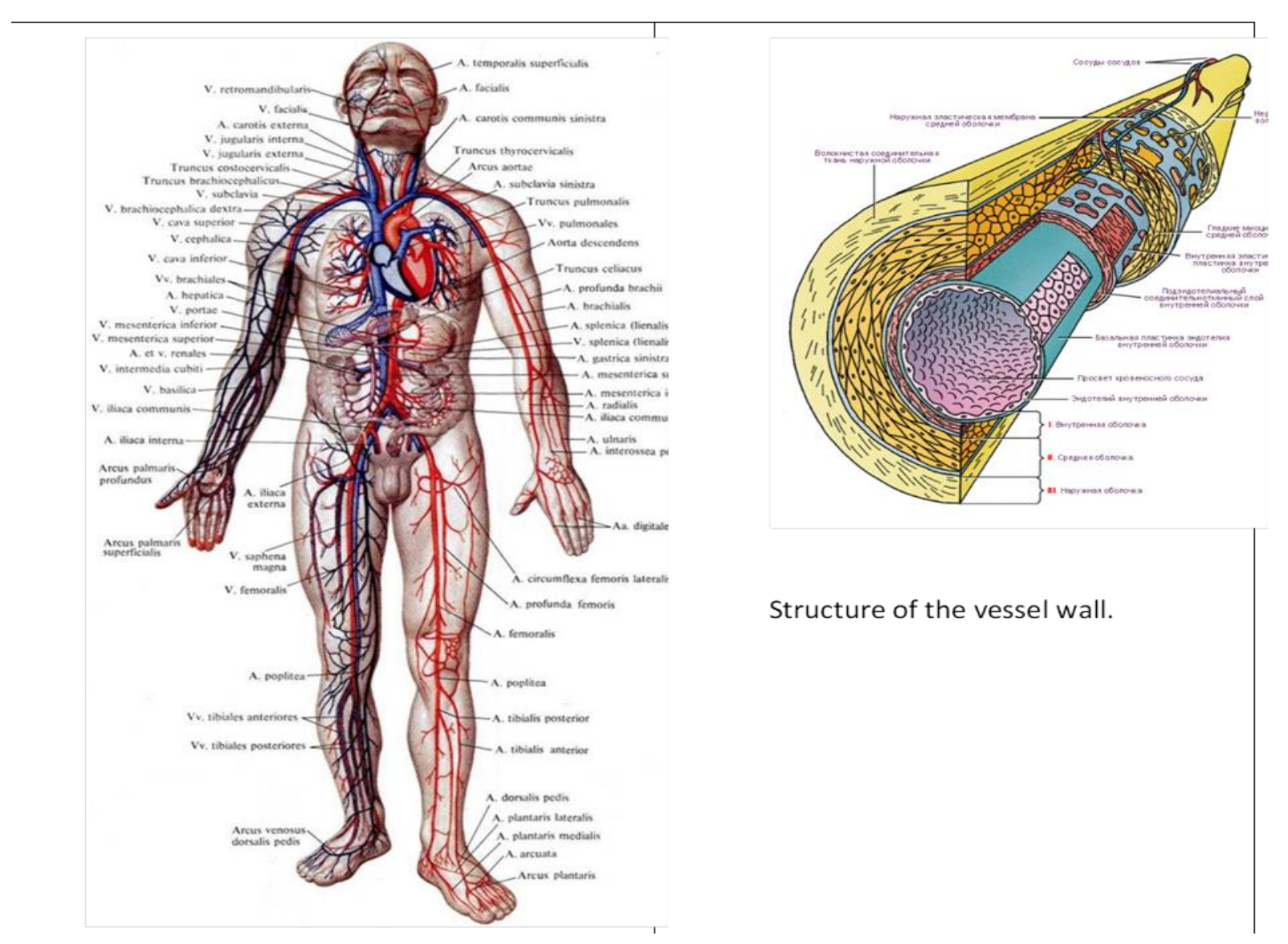

Figure 15 Venous and arterial systems.

The cardiovascular, nervous and lymphatic systems can be light guides and evanescent (attenuated) waves are also formed around them perpendicular to the outer surface of vessels, capillaries, and nerve roots when they are exposed to electromagnetic waves on any part of the skin surface, i.e. the integral organism enters the operation mode of the "scanning tunneling microscope", so evanescent IR spectroscopy of disturbed full reflection when touching the IR fiber is unique, not-damaging, not requiring special skin preparation -"blood spectrum biopsy", which reflects the entire information picture of the body at the atomic level. (A new advanced trend in Medicine). [12,13,14]

An international team of biologists discovered a natural compound that slowed ageing in healthy mice. The study was published in the Cell Metabolism Journal and EurekAlert issue (28.10.2016). Scientists believe that the deficiency of NAD (nicotinamide adenine dinucleotide) in the body is manifested with age. Thanks to NMN (nicotinamide mononucleotide), researchers were able to significantly slow down the physiological decline in ageing mice, as the level of metabolism in these animals was almost equal to that peculiar for young population [20,21].

Scientists believe that such impressive results are relevant for human beings as well.

Testing NMN containing drugs in humans has already begun in Japan. Scientists were able to use the NMN nucleotide, which is involved in the energy exchange of cells for the synthesis of the coenzyme NAD in animals' organisms. It is not possible to directly inject NAD into animals. NAD synthesis in the body slows down with age due to DNA damage. Experiments on mice showed that water-soluble NMN is absorbed into the blood within three minutes and later converted into NAD in tissues. Experts note that taking NMN by young mice has no effect. This compound (NMN), as scientists note, is found in a number of foods, in particular, broccoli, cabbage, cucumbers and avocado. The coenzymes NAD and NADP regulate the metabolism [20,21,22].

NADP is the reduced form of NAD and takes on the hydrogen and electrons of the oxidized compound and transfers them to other substances.

Research conducted by scientists from the University of Washington showed that the substance NMN activates genes responsible for the production of certain proteins and sirtuins. The presence of these proteins in an increased 
concentration in the bodies of experimental rodents caused a significant slowdown in the age-related degradation of vision and metabolic processes in their bodies [21,22,23].

It is also worth noting that modern ideas about the regulation of cellular processes allow us to highlight a nitrogen oxide, which has a multifunctional physiological effect. This free radical can have both activating and inhibiting effects on various metabolic processes occurring in organisms of mammals and humans [20,21].

Intensive study of the biological effect of NO began in the 80's, When R. Furshgott and J. Zawadzki showed that the expansion of blood vessels under the influence of acetylcholine occurs only in the presence of endothelium - epithelial cells lining the inner surface of all vessels.

The substance released by endothelial cells in response not only to acetylcholine, but also to many other external influences resulting in vasodilation (as in the research of Professor K. A. Samoylova) was called "vasodilating endothelial factor". [20,21,24]

Soon later, it was proved that this substance is a NO gas and there are special enzyme systems in cells that are able to synthesize it.

In humans and mammals, nitrogen oxide is mainly formed as a result of the oxidation of the guanidine group of Larginine amino acid with the simultaneous synthesis of another amino acid - citruline - under the influence of NOsynthase enzyme. The enzyme was called a synthase, not a synthetase, because it does not require ATP energy to work. $[1,20,21,23]$

Currently, there are three cell populations that are most studied with respect to synthesis and formation of NO: endothelium of blood vessels, nerve tissue cells (neurons) and macrophages - connective tissue cells with high phagocytic activity. In this regard, there are traditionally three main isoforms of NO-synthases (NOS): neuronal, macrophage, and endothelial (designated respectively as NO-synthase I, II, and III). Neuronal and endothelial isoforms of the enzyme are constantly present in cells and called constitutive, and the second isoform (macrophage) is inducible - the enzyme is synthesized in response to a certain external influence on the cell. [20,22,23]

Based on above, it should be emphasized that the second macrophage isoform (NOS) is synthesized under the influence on the integral organism of PVIP light at a wavelength of 480-3400 nm, according to the research of Professor K. A. Samoilova, there is also a basis to conduct fundamental research on discovering the increase in isoforms I and III under the action of PVIP light, due to the fact that according to our research, there is an instant impact of PVIP light on cardiovascular system and full coverage by the light exposure of the integral organism. $[12,13,14,16]$

Professor K. A. Samoilova claims in her research that the most important role in the stimulating effect of optical radiation on cells and tissues is assigned to two light - absorbing enzyme complexes that have the properties of oxidants-nicotineadenine-dinucletide-phosphate oxidase (NADP-oxidase) and nucleotide-containing biopteroflavoprotein-NO-synthase. Exposed to action of visible and IR light, these enzymes localized in the cell membrane are activated and, using the ambient oxygen, produce its active forms (ROI) - superoxidanion, hydrogen peroxide, hydroxyl radical and nitrogen oxide (NO). These highly reactive molecules conduct a light signal from the surface of the irradiated cell to its nucleus, affecting specialized intracellular mechanisms for conducting the activation signal.

It has already been established that the formation of nitrogen oxide - NO-in the systemic circulation is the most important mechanism for such effects of visible and IR light as dilation of blood vessels and platelet disaggregation, failing which phototherapy could hardly be highly effective. [1]

Before proceeding to considering specific examples of the biological activity of NO in humans and animals, we should once again point out the multifunctional nature of its action, which can not be reduced only to "positive" or only to "negative" effects. [20,21,22]

The biological response to NO is largely determined by the conditions of its generation - where, when, and in what quantity this compound is produced. [19,23]

It is worth noting that to date, the localization, distribution of NO-synthase, non-enzymatic formation of nitrogen oxide, participation of NO in the regulation of the nervous system, in protective immunological reactions, participation of NO in the Central Nervous System (CNS), the role of nitrogen oxide as a regulator of cellular processes in the formation of multiple organ failure are underexplored. 
Based on the above, it should be argued that there is a need to expand basic research on the basis of an academic research Institute with the involvement of specialists in physics, chemistry, cytology, physiology, biology and optical physics to create a methodology with the subsequent development of the subject and program on slowing down the natural ageing of a living organism.

In this regard, it should be considered that the results of the research of Professor K. A. Samoilova, published in the Materials of the scientific and practical conference "New trends in use of light therapy "Bioptron", Moscow, Yekaterinburg, April 2003, are the first data in the World on this problem.

The above concepts were confirmed with a high statistical accuracy by example of a new rehabilitation technology: "Underwater horizontal traction of the spine with underwater phototherapy" to solve an essential medical and social problem: treatment of patients with degenerative and dystrophic lesions of the lumbosacral spine with herniated protrusions of intervertebral discs, being one of the most common neurological pathologies worldwide.

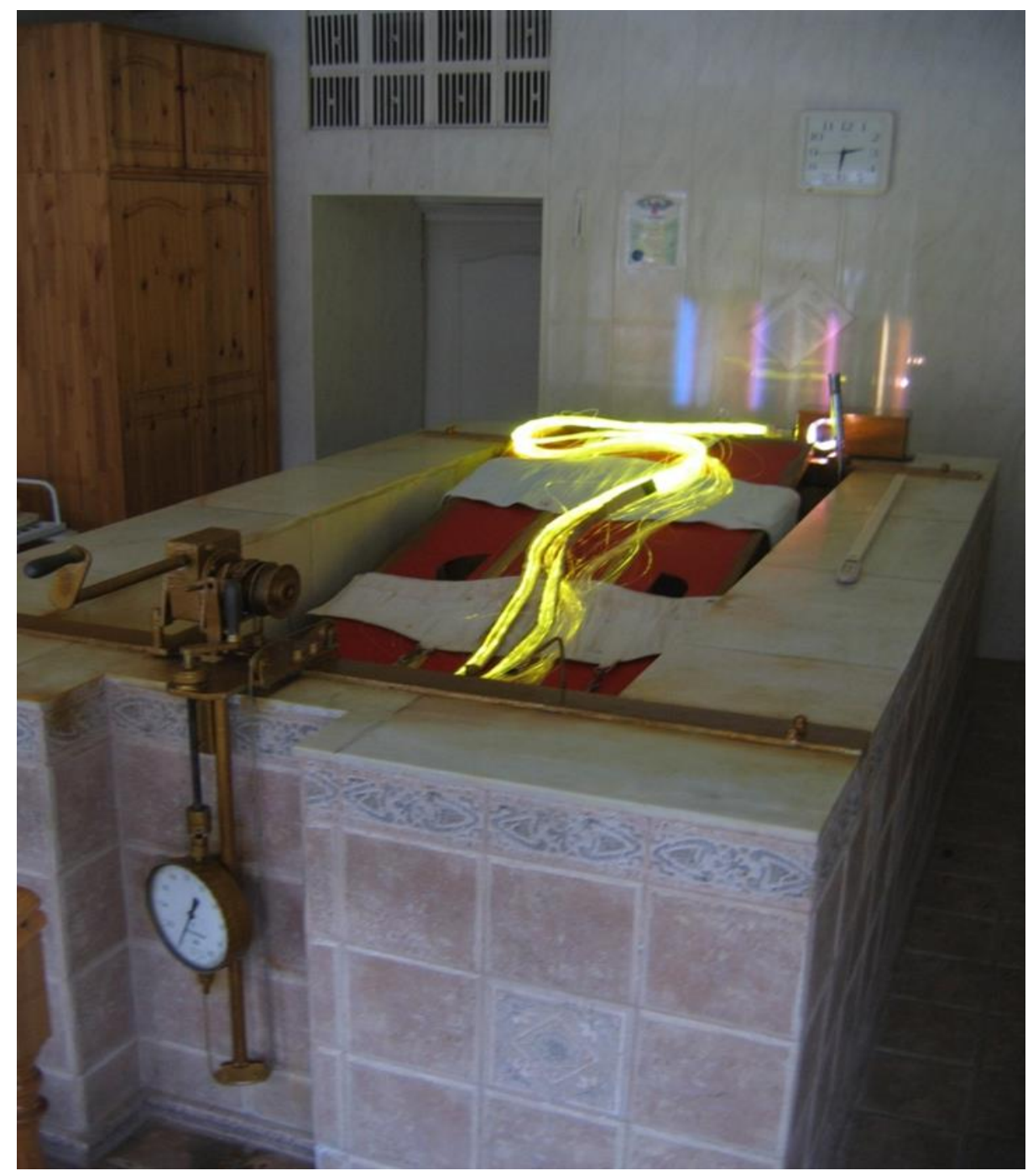

Figure 16 Underwater horizontal spine traction with underwater phototherapy through a fiber optic cable. 
At the present stage of development of rehabilitation medicine, a wide inventory of medicinal and non-medicinal methods (physiotherapy, traction and manual therapy, reflexology, therapeutic physical training), as well as surgical (operative) treatment is used for the treatment of this category of patients.

However, it is not possible to achieve the desired therapeutic effect from the applied methods, due to the ineffectiveness of their combined use $[3,4,5,6]$.

The least effect was received by the patients treated with the first method (control group) of drug therapy: improvement (no pain syndrome with the onset of persistent remission) was observed at $45.7 \%$ of patients, no changes (no clinical dynamics) - at $34.3 \%$ and deterioration (negative clinical dynamics) at $20 \%$.

The results of therapy of patients of the second control group treated with the second method: combination of drugs with physical therapy is slightly higher than in patients of the first control group, namely: improvement (no pain syndrome with the onset of persistent remission) was 54.1\%, no changes (no clinical dynamics) - 30.6\% and deterioration (negative clinical dynamics) - 15.3\%.

It should be noted that the efficacy of treatment methods 3,4 and 5 , where one of the components of treatment is underwater spinal traction or underwater phototherapy, is significantly higher than in the first and second control groups, by $25-34 \%, 33-41 \%$ and $43-51 \%$ respectively.

It should also be noted that in the fifth group ( $\mathrm{n}=128)$, improvement (no pain syndrome with the onset of stable remission) was observed in the first age group of patients aged 17 to 30 yerars, the fourth age group of patients aged 51 to 60 years, with the protrusion of HNP Z4-S1 into the spinal canal in cases of up to $5 \mathrm{~mm}$ and from $5 \mathrm{~mm}$ to $13 \mathrm{~mm}$, $100 \%$ improvement was observed in patients of the second age group aged 31 to 40 years with the protrusion of HNP Z4-S1 into the spinal canal from $5 \mathrm{~mm}$ to $13 \mathrm{~mm}-92.3 \%$.

In three groups $(3,4$ and 5), the largest share of patients without changes (no clinical dynamics) was found in the third group - $20.3 \%$, and among the age groups, the largest share was found in patients of the third age group 41 to $50-7.8 \%$.

The smallest share of patients without changes (no clinical dynamics) was represented by the patients of the fifth group $(\mathrm{n}=128)-2.4 \%$, and among the age groups, the largest share was also found in patients of the third age group aged 41 to 50 years $-1.6 \%$.

As a result of comparisons of two control groups ( $\mathrm{I}-\mathrm{n}=175$ and II-t=157) by age groups, it was found that the deterioration (negative clinical dynamics) was detected in the fourth age group of patients aged 51 to 60 years of the first control group $n=175-6.8 \%$, and the smallest share was represented by patients of the first age group of patients aged 17 to 30 years $-2.3 \%$ of the second control group $n=157$.

The analysis of long-term results of treatment of patients with dorsopathy, osteochondrosis of the lumbosacral spine with protrusion into the spinal canal of HNP L4-S1 up to $5 \mathrm{~mm}$ and from $5 \mathrm{~mm}$ to $13 \mathrm{~mm}$ for three years is presented in table 25.

It follows that the lowest incidence of relapse of severe pain syndrome was observed in groups 3,4 and $5-20 \%$ in comparison with control groups 1 and $2-80 \%$.

It is worth noting that the inclusion of underwater traction of the lumbosacral spine and underwater phototherapy into the treatment regimen results in a lower incidence of pain relapse compared to the control groups treated with drugs only (first control group $\mathrm{n}=175$ ) and a combination of drugs and physiotherapy (second control group $\mathrm{n}=157$ ).

In other words, "the joint use of underwater horizontal spine traction with underwater phototherapy via fiber optic cable" contributes to restoration of impaired functions in $96-98 \%$ of patients with degenerative-dystrophic lesions of the spine, due to the regression of herniated protrusion of intervertebral discs by $30-50 \%$ and anti-inflammatory antioxidant effect of underwater phototherapy, but to date, such a regimen has not been used anywhere in the world for this pathology.

At the same time, repeated treatment during 5-7 years was undergone only by $0.5 \%$ of patients, and the pain passed in all patients after the first procedure. This is due to the unique combination of underwater horizontal spine traction with underwater phototherapy (yellow light with infrared light at wavelength of 480-3400 nm) via a fiber optic cable [11]. 
A high percentage of positive treatment outcomes, both immediately after the course of therapy and in the long term, indicates the high efficacy of the developed complex of rehabilitation medicine of underwater lumbosacral spine traction with underwater phototherapy.

The reliability of treatment outcomes is supported by the use of modern highly sensitive informative methods for assessing the condition of intervertebral discs (MRI and CT) and pathological morphological processes using blood plasma spectroscopy, blood spectrum biopsy from the skin surface for the first time in world healthcare practice. [1,14]

It should be noted that the presented rehabilitative technology surpasses all existing pharmaceutical products, nondrug treatment methods and occupies a well-deserved leading place in the primary prevention of degenerative and dystrophic spinal lesions in adolescents, sportsmen, drivers of road, railway and other vehicles with a driving time of more than 60 minutes per day.

The high efficacy of anti-inflammatory immunomodulatory, antiviral and antitumor effects of polarized light at 480$3400 \mathrm{~nm}$ was confirmed by fundamental research of Russian and foreign scientists: Doctor of Biology, Professor K. A. Samoilova. Institute of Cytology of the Russian Academy of Sciences, Saint Petersburg, 2003; Doctor of Medicine, Professor L. N. Gerasimova, Burn Center of the N. V. Sklifosovsky Research Institute of Emergency Medicine, Moscow; Professor M. Lentz, Oxford University, UK; Professor L. Medinica, Institute of Dermatovenerology of the Clinical Center, University of Belgrade, Yugoslavia. [11,16]

A peculiarity of phototherapy performed using PVIP light is a very rapid "disappearance" of pro-inflammatory cytokines - tumor necrosis factor (TNF- $\alpha$ ), interleukins-IL-6, IL-2 and IL-12 from the circulating blood, which is recorded just within 30 minutes after the first irradiation. So, at the initial increased content of TNF- $\alpha$, it drops by 30 times, IL- 8 - by 4-6 times, IL-2 - by 4-10 times and IL-12 - by 12 times (by the end of the treatment course). At the same time, the levels in plasma of anti-inflammatory cytokines - IL-10 and transforming growth factor - TGF- $\beta 1$ increase, as well as a rapid six-fold increase in the blood of the most important immunomodulator - interferon- $\gamma$ (IFN- $\gamma$ ) even at its initial normal levels. The most important function of this cytokine is to activate cellular immunity (functional state of monocytes, macrophages, natural killers, and cytotoxic T-lymphocytes), which primarily increases the body's antiviral and antitumor resistance [24].

Experience with PVIP irradiation of blood in vitro gives reason to associate all the described effects with the direct action of light on the blood. In experiments of all designs, light not just stimulates, but regulates the cytokines levels.

Also, there is also direct contact in vivo with the cardiovascular system (blood) through perspiration.

This is an instantaneous effect on the cellular, supramolecular (atomic) level $[13,14,16]$.

In this regard, there is reason to assert that the system of underwater horizontal spine traction with underwater phototherapy, for the primary disease prevention in adolescents, sportsmen and improving the quality of life of patients with degenerative and dystrophic lesions of the spine, deserves to be promptly introduced into the practice of rehabilitation centers, medical and sports dispensaries, health resorts, departments of therapeutic physical training of healthcare institutions and boarding houses.

A human being is a particle of Nature, and all biological processes proceed in him or her in accordance with a strict "program", in the strictest hierarchy from elementary particles to complex structures $[12,13]$.

There are no two identical elementary particles in nature, hence the supramolecular formations consisting of them up to the Cosmos itself. As exemplified by a living organism in any system (muscular, circulatory, nervous, cardiovascular, etc.), there are no two identical cells either in content or in form, because there would be no muscle contraction, impulse transmission, intracellular and intercellular movement, i.e. the complete absence of visible life activity, i.e. movement with super-low speeds at the supramolecular level, but our cognition of the same is limited by the range of human knowledge $[13,14]$.

The intracellular structure of any system is unique, so the intracellular energy is. Therefore a difference of intercellular energy exists as a source of constant endless movement with the huge endless range of speeds, which creates a cyclical nature of processes of "matter-energy" transition from one state to the next ones. This should be considered by a specialist physician, when prescribing drug therapy to patients, otherwise it may result in various complications and addiction to drugs (disorders of organs and systems functions, development of drug addiction, allergies to drugs) $[13,14,25]$. 
It is known that "the concept of radiation hormesis was introduced to biology in the 80's and, as homeopathy postulated, while large doses of radiation have adverse effects on living organisms - inhibit cell division, growth and development, then small doses stimulate almost all physiological processes." The effects associated with the manifestation of the stimulating effect of low doses of radiation were called radiation hormesis. [26]

In this regard, there is a reason to believe that any physical factor affects the body through liquid perspiration from the skin surface. The cardiovascular system instantly connects the physical factor to all organs and systems at the supramolecular level. The same instantaneous connection is carried out by the "tunnel effect", but the current level of technological development does not allow to differentiate them.

According to Einstein's famous formula stating the "mass-energy equivalence" and thus justifying the process of mutual transformation of mass and energy $\left(\mathrm{E}=\mathrm{m} c^{2}\right)$, the real primary quantum touch of pathological cells with the physical factor selected for treatment is completed.

Weak physical effects are more selective and require a very fine adjustment of parameters of the same, in order to implement an interaction contributing to restoration of the disturbed specific biological process [13,14].

A selective, correct method of a therapeutic physical factor application for a specific intermediate state of the integral organism in the course of its cyclic development at pathology provides for the rejection of use of high-energy parameters of the selected physical factor. This is necessary to prevent the simultaneous destruction of intermediate cyclic states of weakened morphological supramolecular formations of the body and strong pathological supramolecular formations, which is a gross interference with the biological cyclic processes of the body.

This can lead to complete or partial irreversible states of organs and systems of the integral organism. In this regard, it should be argued that the application of "established medicamentous standards for diseases" for all patients with the same diagnosis is anti-scientific and extremely dangerous.

Due to the fact that any disease is unique for each patient: etiology, pathogenesis, clinical findings, disease outcome, consequences for the integral organism in the future, because even two identical pathogens of any disease do not exist in Nature.

\section{Conclusion}

Development of a system of rehabilitation treatment of patients with degenerative and dystrophic lesions of the lumbosacral spine with herniated protrusions of intervertebral discs is an challenging medical and social problem (this pathology is one of the most common neurological diseases in the world and, especially, in Russia).

The combined use of underwater horizontal spine traction with underwater phototherapy can contribute to restoration of impaired functions in patients with degenerative and dystrophic lesions of the spine, but to date, such a complex has not been used for treatment of this pathology.

From the point of view of rehabilitation medicine, this work implements a comprehensive therapy of herniated protrusions of the intervertebral discs of the lumbosacral spine through a new system of rehabilitation treatment by underwater horizontal spine traction and underwater phototherapy.

The therapeutic effect on the body of polychromatic visible and infrared polarized light through the water environment, which may be determined by its activating effect on water, was proved for the first time under experimental conditions. The author a) showed a remarkable regression of herniated protrusion of the intervertebral disc of the lumbosacral spine, when using the developed system of rehabilitation treatment. b) substantiated the expediency of using and a) proved a high clinical efficacy of the combined use of underwater horizontal spine traction and underwater phototherapy for treatment of degenerative and dystrophic spine lesions.

In a comparative perspective, a high efficacy of rehabilitation technology applied according to the author's method was established in comparison both with drug therapy and combination with other known methods of physiotherapy and drug treatment.

The results of experimental and clinical data confirm the clear advantage of the physical factors use over drug therapy in case of pathology of the musculoskeletal system. 
Therefore, "Physiotherapy science" should take its rightful place among the leading scientific disciplines of the world medical science.

\section{References}

[1] Bertram G, Katzung MD. PhD Translation from English edited by Doctor of Medicine, Prof. Zvartau E. E. Basic and clinical pharmacology. 1998; 1(2).

[2] Volkov ES, Vlyalko VI, Volkov ES, Vlyalko VI. Electricity at service of health. K.: Zdorovye. 1985; 446.

[3] Bogolyubov VM, Ponomarenko GN. General Physiotherapy. 2nd Revised edition. M., Saint Petersburg: SPP. 1997; 480 .

[4] Clinical Physiotherapy / edited by Orzheshkovskiy V. V., K.: Zdorovye. 1985; 446.

[5] Klyachkin LM, Vinogradova MN. Physiotherapy M.: Meditsina. 1982; 272.

[6] Ulaschik VS. Analytical reviews of general physiotherapy. Minsk. Nauka i tekhnika Publ. 1994; 198.

[7] Aldersons AA. Mechanism of electrodermal reactions. Riga. 1985.

[8] Garyaev PP. Wave genetic code. Moscow.1997.

[9] Jean-Marie Lehn. Supramolecular Chemistry. Novosibirsk, Nauka. 1998.

[10] Andryushin EA, Andryushin EA. Power of nanotechnologies: science \& business/Andryushin E.A. - B.M.: Successes of Physics. 2007; 155.

[11] Bitsoev VD. System of rehabilitation treatment of degenerative and dystrophic lesions of spinal cord: Abstract of thesis of Doctor of Medicine. - Moscow. 2012; 40.

[12] Bitsoev VD. New line in research of a role of interaction between the organism and physical factors in comprehensive therapy of patients. Moscow. 2015.

[13] Bitsoev VD. The Application of Corrective Underwater Spinal Traction with Underwater Phototherapy to the Spine Pathology. Clinical Medicine Research. November 2015; 4(6), 204-213.

[14] Bitsoev VD. New approach in investigating the role of interaction between AN ORGANISM and physical factors in complex patient therapy. - Open Access Library Journal. March 2016.

[15] Batanov GM, Bolotovsky BM, Grigoryan SS, Kossyi IA, Sokolov IV. In memory of G. A. Askarian, Moscow: Znak. 1998; 376.

[16] Bitsoev VD. Blood Spectrum Biopsy as Screening Diagnostics of Health. - American Scientific Journal. - №7. 2016.

[17] Oldenburg Michael. "Photon Upconversion at Crystalline Organic-Organic Heterojunctions".- Advanced Materials. August 2016; 95.

[18] Goltsman GN. Josephson effects in superconductors. // Sorovsky Educational Journal. 2000; 6(4): 96-202 .

[19] Brandt NB. Superconductivity / Sorovsky Educational Journal. 1996; 1: 100-107.

[20] Ryabov GA, Azizov Yu M. "The role of nitrogen oxide as a regulator of cellular processes in the formation of multiple organ failure". Educational and Research Center of the Medical Center at Administration of the President of the Russian Federation, Moscow.

[21] Sosunov AA. Nitrogen oxide as an intercellular mediator. N.P. Ogarev Mordovian State University, Saransk. 2000.

[22] Malkoch AV, Maydannik VG, Kurbanova EG. The physiological role of nitrogen oxide in organism (Part 1).

[23] Alberts A, Bray D, Lewis R. Molecular biology of the cell: in 3 vols.: Translation from English 2nd ed. M.: Mir. 1994.

[24] Samoylova KA. Mechanism of anti-inflammatory, immunomodulating, wound healing and normalizing the metabolism light of the device "BIOPTRON", Materials of Scientific and Practical Conference, Moscow, Yekaterinburg. April 2003.

[25] Einstein A. Collection of research works IV Articles, reviews, letters. Evolution of physics. - Nauka publishing house, Moscow. 1967. 